\title{
Synchronous behavior of two coupled electronic neurons
}

\author{
R. D. Pinto, ${ }^{1}$ P. Varona, ${ }^{1,2}$ A. R. Volkovskii, ${ }^{1}$ A. Szücs, ${ }^{1}$ Henry D. I. Abarbanel, ${ }^{1,3}$ and M. I. Rabinovich ${ }^{1}$ \\ ${ }^{1}$ Institute for Nonlinear Science, University of California, San Diego, La Jolla, California 92093-0402 \\ ${ }^{2}$ GNB, Departamento Ingeniería Informática, Universidad Autónoma de Madrid, 28049 Madrid, Spain \\ ${ }^{3}$ Department of Physics and Marine Physical Laboratory, Scripps Institution of Oceanography, \\ University of California, San Diego, La Jolla, California 92093-0402
}

(Received 12 January 2000)

\begin{abstract}
We report on experimental studies of synchronization phenomena in a pair of analog electronic neurons (ENs). The ENs were designed to reproduce the observed membrane voltage oscillations of isolated biological neurons from the stomatogastric ganglion of the California spiny lobster Panulirus interruptus. The ENs are simple analog circuits which integrate four-dimensional differential equations representing fast and slow subcellular mechanisms that produce the characteristic regular/chaotic spiking-bursting behavior of these cells. In this paper we study their dynamical behavior as we couple them in the same configurations as we have done for their counterpart biological neurons. The interconnections we use for these neural oscillators are both direct electrical connections and excitatory and inhibitory chemical connections: each realized by analog circuitry and suggested by biological examples. We provide here quantitative evidence that the ENs and the biological neurons behave similarly when coupled in the same manner. They each display well defined bifurcations in their mutual synchronization and regularization. We report briefly on an experiment on coupled biological neurons and four-dimensional ENs, which provides further ground for testing the validity of our numerical and electronic models of individual neural behavior. Our experiments as a whole present interesting new examples of regularization and synchronization in coupled nonlinear oscillators.
\end{abstract}

PACS number(s): 05.45.Xt, 84.35.+i, 87.80.-y, 87.18.Sn

\section{INTRODUCTION}

Synchronization of nonlinear oscillators is widely studied in physical and biological systems [1,2] for underlying interests ranging from novel communications strategies $[3,4]$ to understanding how large and small neural assemblies efficiently and sensitively achieve desired functional goals [5]. The analysis of biological systems may, beyond their intrinsic interest, often provide physicists with novel dynamical systems possessing interesting properties in their component oscillators or in the nature of the interconnections.

We have presented our analysis of the experimental synchronization of two biological neurons as the electrical coupling between them is changed in sign and magnitude [6]. Subsequent to that analysis we have developed computer simulations of the dynamics of the neurons which are based on conductance based Hodgkin-Huxley (HH) [7] neuron models. These numerical simulations quantitatively reproduced the observations in the laboratory $[6,8-10]$.

The study of isolated neurons from the stomatogastric ganglion (STG) of the California spiny lobster Panulirus interruptus using tools of nonlinear time series analysis $[11,12]$ shows that the number of active degrees of freedom in their membrane potential oscillations typically ranges from three to five [2]. The appearance of low dimensional dynamics in this biological system led us to develop models of its action potential activity, which are substantially simpler than the HH models we and others [8] have used to describe these systems. We adopted the framework established by Hindmarsh and Rose (HR) [13] in which the complicated current-voltage relationships of the conductance based models are replaced by polynomials in the dynamical variables, and the coefficients in the polynomials are estimated by analyzing the observed current/voltage curves for the neurons under study.
Building on biological experiments and on numerical analysis of models for the oscillations of isolated neurons, we have constructed low-dimensional analog electronic neurons (EN's) whose properties are designed to emulate the membrane voltage characteristics of the individual neurons. Using these simple, low dimensional EN's we report here their synchronization and regularization properties, first when they are coupled electrically as the sign and magnitude of the coupling is varied, and then when they are coupled by excitatory and inhibitory chemical synapses. We have also studied the behavior of a hybrid system, i.e., one EN and one biological neuron coupled electrically. As our models were developed on data acquired from biological neurons in synaptic isolation, the results we present here on pairs of interacting ENs and hybrid systems serve to provide further confirmation of the properties of those model neurons, numerical and analog.

\section{ELECTRONIC NEURON MODEL}

We have studied and built three-dimensional (3D) and four-dimensional (4D) models of HR type having the form

$$
\begin{gathered}
\frac{d x(t)}{d t}=a y(t)+b x^{2}(t)-c x^{3}(t)-d z(t)+I, \\
\frac{d y(t)}{d t}=e-f x^{2}(t)-y(t)-g w(t), \\
\frac{d z(t)}{d t}=\mu(-z(t)+S(x(t)+h)), \\
\frac{d w(t)}{d t}=\nu(-k w(t)+r(y(t)+l)),
\end{gathered}
$$



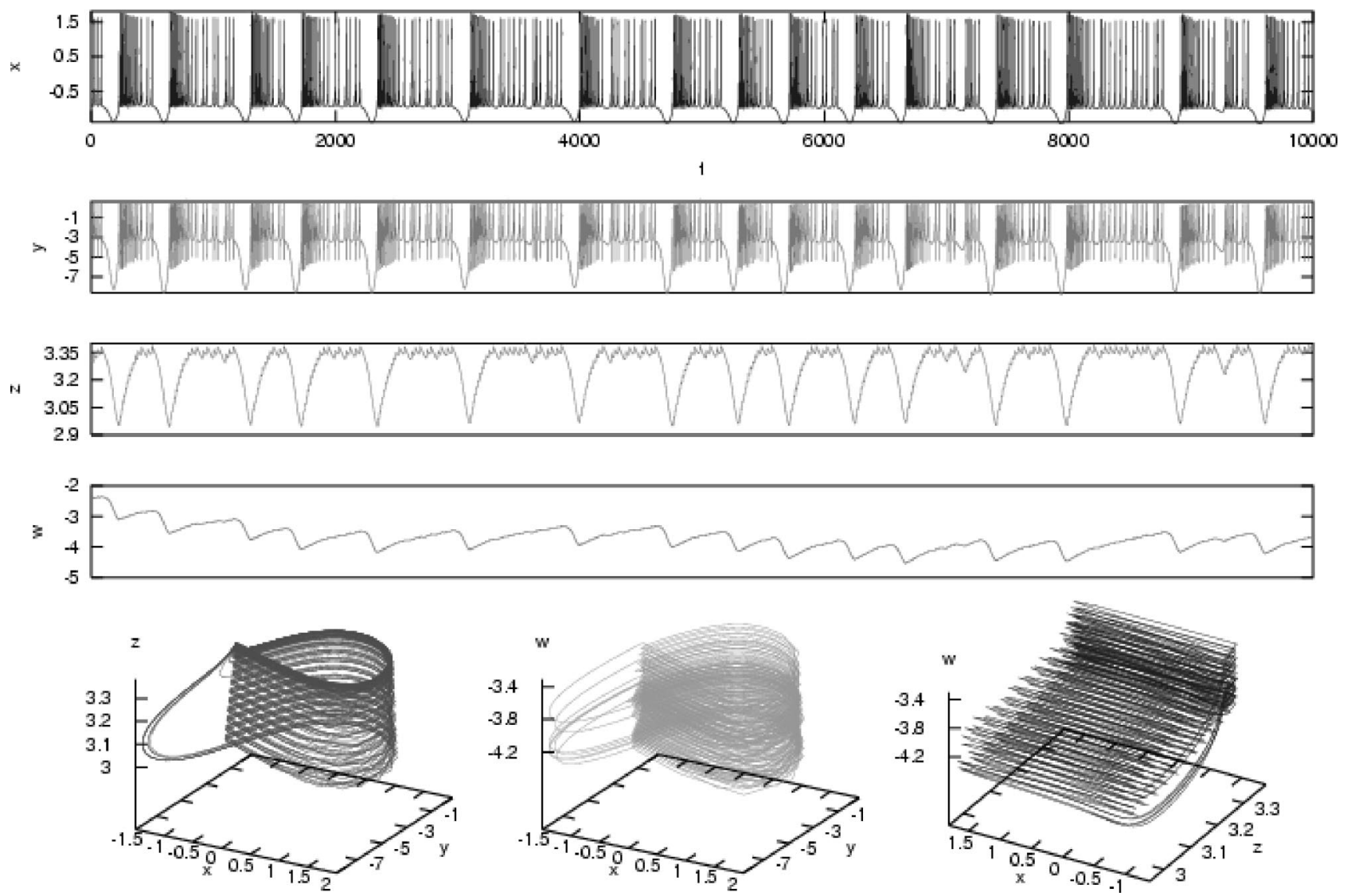

FIG. 1. Time series of the dynamical variables $x(t), y(t), z(t), w(t)$ of our 4D HR model, Eq. (1), and various 3D projections $(x(t), y(t), z(t)),(x(t), y(t), w(t))$ and $(x(t), z(t), w(t))$ of the $4 \mathrm{D}$ phase space orbits. Units are dimensionless in the numerical simulations

where $a, b, c, d, I, e, f, g, \mu, S, h, \nu, k, r$, and $l$ are the constants that embody the underlying current and conductance based dynamics in this polynomial representation of the neural dynamics. $x(t)$ is the membrane voltage in the model, $y(t)$ represents a "fast" current in the ion dynamics, and we choose $\mu \ll 1$, so $z(t)$ is a "slow" current. Taken alone the first three equations of the model can reproduce several modes of spiking-bursting activity observed in STG cells. The first three equations were used in analog realization for our earlier experiments with 3D ENs [14].

The equation for $w(t)$ represents an even slower dynamical process $(\nu<\mu \ll 1)$, and it is included because a slow process, such as the calcium exchange between intracellular stores and the cytoplasm, was found to be required in $\mathrm{HH}$ modeling [8] to fully reproduce the observed chaotic oscillations of STG neurons. Both the 3D and 4D models have regions of chaotic behavior, but the $4 \mathrm{D}$ model has much larger regions in parameter space where chaos occurs, presumably for many of the same reasons the calcium dynamics gives rise to chaos in $\mathrm{HH}$ modeling. The calcium dynamics is an additional degree of freedom with a time constant three times slower than the characteristic bursting times. In addition, the 4D model allows a better adjustment of the behavior of the EN when connecting it to living neurons. In particular, we can adjust several details of the shape of the spiking-bursting activity, for example the width of the bursts, before setting the model neuron into the chaotic regime.

In our analog circuit realization of the EN model we used $a=1, b=3, c=1, d=0.99, I=3.024, e=1.01, f=5.0128$, $g=0.0278, \mu=0.0021, S=3.966, h=1.605, \nu=0.0009, k$ $=0.9573, r=3.0$, and $l=1.619$. The implementation of these constants in analog circuits always has about $5 \%$ tolerance in the components. The main parameters we used in controlling the modes of spiking and bursting activity of the model are the dc external current $I$ and the time constants $\mu$ and $\nu$ of the slow variables.

Figure 1 shows a chaotic time series of the four variables using the parameters above. Note how $w$ modulates the length of the bursts in $x$. Each local minimum in the global oscillations of $w$ coincides with a short burst period. The complexity achieved by the addition of $w$ can be observed in the projections of $(x, y, z, w)$ space to various 3D spaces, $(x, y, z),(x, y, w)$, and $(x, z, w)$, respectively, as shown in Fig. 1.

Table I presents the Lyapunov exponents $\lambda_{i}$ calculated from the vector field [15] of Eq. (1) for the 3D and 4D EN's. A positive Lyapunov exponent is present in both models, indicating conclusively that they are oscillating chaotically. From this spectrum of Lyapunov exponents, we can evaluate the Lyapunov dimension $D_{L}$ which is an estimate of the fractal dimension of the strange attractor for the EN's [11]. The Lyapunov dimension is defined by finding that number of Lyapunov exponents $\lambda_{i}$ satisfying

$$
\sum_{i=1}^{N} \lambda_{i}>0
$$


TABLE I. Lyapunov exponents $\lambda_{i}$ and Lyapunov dimension $D_{L}$ calculated from the vector field [Eq. (1)] for the 3D and 4D electronic neuron models. As a reminder to the reader: the sum of all Lyapunov exponents must be negative, and this is so for our results. Also, one Lyapunov exponent must be 0 as we are dealing with a differential equation.

\begin{tabular}{cccccc}
\hline \hline Model: & $\lambda_{1}$ & $\lambda_{2}$ & $\lambda_{3}$ & $\lambda_{4}$ & $D_{L}$ \\
\hline 3D & 0.010 & 0.000 & -7.752 & & 2.001 \\
4D & 0.004 & 0.000 & -0.001 & -8.034 & 3.000 \\
\hline \hline
\end{tabular}

while

$$
\sum_{i=1}^{N+1} \lambda_{i}<0
$$

Then $D_{L}$ is defined as

$$
D_{L}=N+\frac{\sum_{i=1}^{N} \lambda_{i}}{\left|\lambda_{N+1}\right|} .
$$

$D_{L}$ for each EN is displayed in the last column of Table I.

\section{ANALOG IMPLEMENTATION OF THE EN'S}

We designed and built an analog electronic circuit that integrates Eq. (1). We chose to build an analog device instead of using numerical integration of the mathematical model on the CPU of a PC or on a DSP board because digital integration of these equations is a slow procedure associated with the three different time scales in the model. Furthermore, a digital version of an EN requires digital to analog and analog to digital converters to connect the model to biological cells. Analog circuits are small, simple, and inexpensive devices; it is easy to connect them to a biological cell, as we discuss below (see also Ref. [14]). In a practical sense nearly an unlimited number of them can work together in real-time experiments. Finally, looking ahead to the construction of real-time networks of large numbers of these neurons, analog implementation is a necessity.

The block diagram of the analog circuit we use to represent the 3D and the 4D EN's is shown in Fig. 2. It includes four integrators indicated by $\int d t$, two multipliers, two adders, and two inverters. We used off-the-shelf general purpose operational amplifiers (National Instruments Model TL082) to build the integrators, adder and inverter, and used Analog Devices Model AD633 as analog multipliers. The integrator at the top of the diagram receives all components of $d x(t) / d t$, e.g., $a y(t), b x^{2}(t)$, etc. It has an additional input (called $i n$ ) which can be used for connections with other circuits or neurons. The integrators invert the sign of their input, so the output signal will be $-x(t)$ multiplied by a time constant $\tau$ chosen to make the EN oscillate on the same time scale as the biological neurons. The signal $-x(t)$ is used to generate the nonlinear functions $x^{2}(t)$ and $x^{3}(t)$ and these values go to the second and third integrators. Similarly, the other integrators generate voltages proportional to $y(t),-z(t)$, and $w(t)$. A renormalization was made in the rest of the time constants in the circuit to make $\tau=1$.

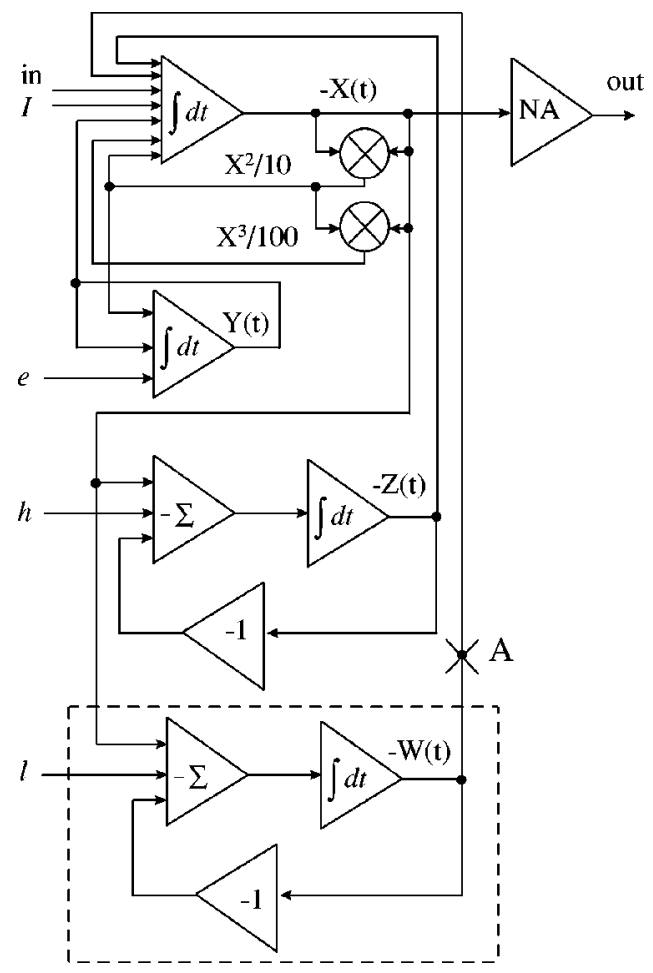

FIG. 2. Block diagram for the 4D HR neuron used in our experiments. These neurons were designed to replicate the behavior of individual, isolated neurons from the lobster STG. In our experiments they were coupled electrically as well as via an electronic implementation of inhibitory and excitatory chemical synapses.

Note that this rescaling is responsible for the different amplitudes in the numerical (Fig. 1) and analog (Figs. 3, 5, 6, 8, 9-no longer dimensionless) experiments.

This circuit design allows us to easily switch from a 3D to a 4D model of the neuron. We can connect or disconnect one wire, indicated as point A in Fig. 2, to enable or disable the circuit block shown in the rectangle with a dashed outline. In Eq. (1) this corresponds to setting $g=0$ in the $d y(t) / d t$ equation.

The block indicated as NA in Fig. 2 is an adjustable nonlinear amplifier. We use it to rescale and change the shape of the output signal $x(t)$. It can shrink or stretch different parts of the wave form, change the amplitude, and move the trace as a whole up or down. This shape adjustment is particularly important in experiments with groups of biological and electronic neurons interconnected with each other. Living neurons, even taken from the same biological structure, may generate considerably different wave forms. The relative size of spikes and the interburst hyperpolarization is variable from cell to cell. In our circuits we can precisely adjust the wave form of the EN to be very close to that of each biological neuron in our experiments.

Another reason to use circuits with variable wave forms is that it opens up the possibility of studying how the action potential wave forms affect the interactions among the neurons, electronic and biological. Indeed, the ability to vary the details of the wave forms provides an interesting handle on design of biometric circuitry for a variety of applications. 

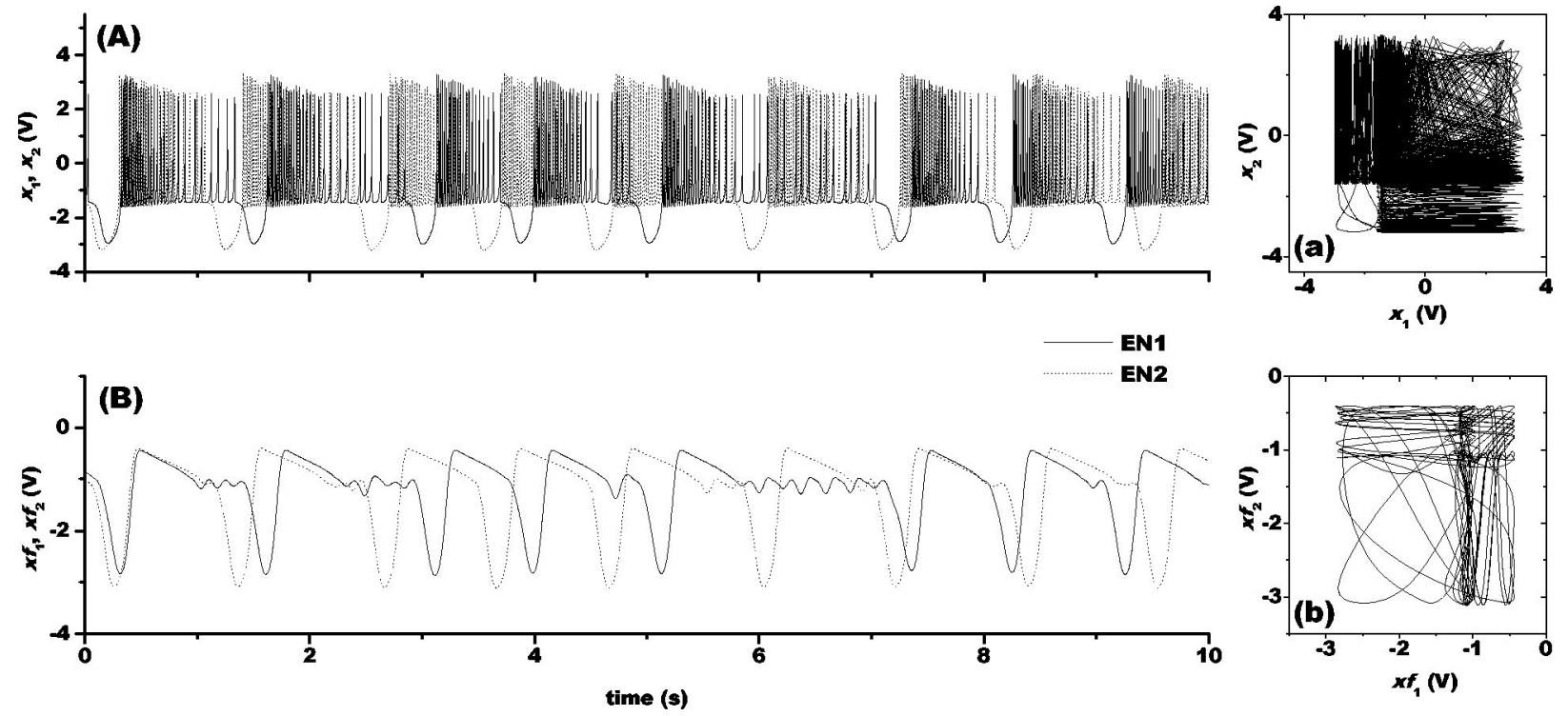

FIG. 3. Regimes of oscillations in two uncoupled electronic neurons (EN's). (A) Time series of the membrane voltages $x_{1}(t), x_{2}(t)$ for the uncoupled EN's. (a) Phase space portraits of $x_{2}(t)$ vs $x_{1}(t)$. (B) Membrane potentials after 20-Hz low-pass filtering to emphasize the bursting behavior. (b) Phase space portraits of filtered membrane potentials $x_{2}^{f}(t)$ vs $x_{1}^{f}(t)$.

\section{SYNAPTIC CONNECTIONS BETWEEN EN's}

In living nervous systems one finds three general types of synaptic connections among neurons [16]: ohmic electrical connections (also called gap junctions) and two types of chemical connections; excitatory and inhibitory. For our studies of the interconnections among EN's and among EN's and biological neurons [14], we built electronic circuits to emulate excitatory and inhibitory synaptic connections as well as the ohmic electrical connections. The STG neural circuits are dominated by inhibitory interconnections and by ohmic electrical connections. We now describe how we implemented each, and then we turn to the results of our synchronization experiments with these network connections.

\section{A. Implementation of the electrical synapses}

We implemented an electrical synapse [17] between the EN's by injecting into one of the neurons $\left(\mathrm{EN}_{1}\right)$ a current proportional to the voltage difference between the two membrane potentials of the EN's and into the other neuron $\left(\mathrm{EN}_{2}\right)$ injecting the same current but with the opposite strength. The current into $\mathrm{EN}_{1}$ is

$$
I_{1}(t)=\frac{G_{E}}{470 \mathrm{k} \Omega}\left(x_{2}(t)-x_{1}(t)\right) ;
$$

while

$$
I_{2}(t)=-I_{1}(t)
$$

We chose the dimensionless synaptic strength $G_{E}$ in the range $G_{E} \in[-1,1]$. Over this range we observed the effects of positive and negative electrical coupling on the spiking and bursting behavior of the EN's. We recorded the electrical voltage signals corresponding to the membrane potentials of the EN's using an analog to digital converter with a sampling rate of $5 \mathrm{kHz}$. For each value of $G_{E}$ we waited at least $40 \mathrm{~s}$ to avoid transient dynamics and then recorded a data series 20 s long.

Natural biological networks do not have negative conductance electrical coupling. Using an active device placed between the neurons we implemented negative electrical couplings in our experiments on two electrically coupled biological neurons as reported in Ref. [6]. To compare the results of our work there with the properties of coupled EN's, we use negative coupling here as well.

\section{B. Implementation of the chemical synapses}

We first implemented mutual chemical synapses between the two EN's using analog circuitry. Here we report on results obtained by using a software implementation of the chemical synapses which allows us to investigate the role of the synaptic time constant $\tau_{s}$. In the analog circuit implementation of the chemical synapses we need to replace a capacitor every time we want to change the time constant, but in the software version this time constant is just a parameter, so it is easier to study the role of these time constant in the software version. In this paper the time constant is fixed, and our observations on the role of a changing time constant will be reported in another paper. The results using a software version of the chemical synapse, and the results using our hardware version were identical.

We used the nonlinear amplifiers to reshape the signals corresponding to the membrane potential of the EN's in such a way that the new signals had amplitudes, spike/burst ratios, and voltage offsets close to the signals generated by living neurons. With these reshaped signals we used new dynamic clamp software [18] to generate in real time the currents corresponding to the graded chemical synapses as described by the first-order kinetics

$$
I_{C}=213 G_{c} S(t)\left(x_{\mathrm{rev}}-x_{\mathrm{post}}\right),
$$




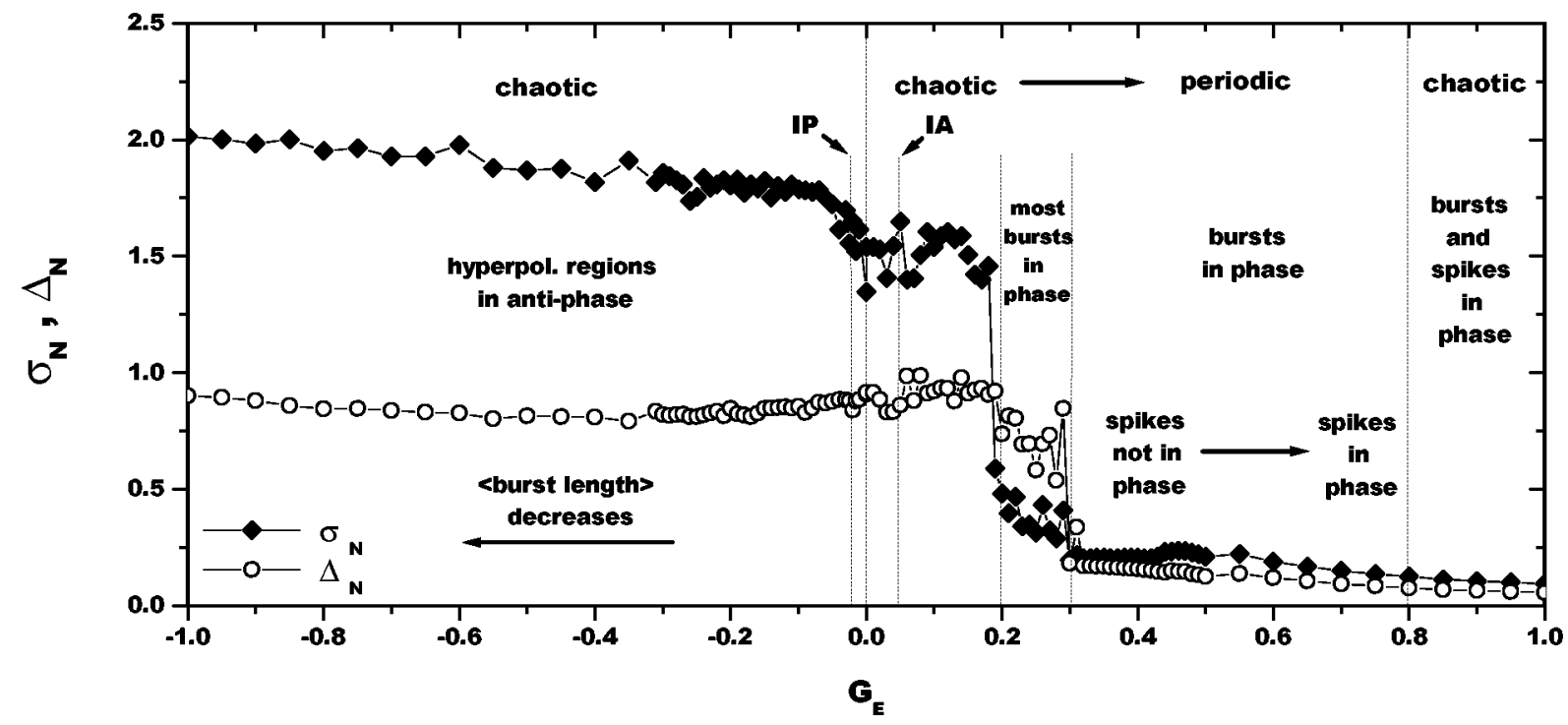

FIG. 4. Normalized standard deviation $\sigma_{N}\left(G_{E}\right)$ and normalized maximal deviation $\Delta_{N}\left(G_{E}\right)$ computed after 20-Hz low-pass filtering the membrane potential of two electrically coupled EN's for different values of the synaptic conductance $G_{E}$. For $G_{E} \gtrsim 0$ the behavior of the EN's is nearly independent and chaotic. The region labeled as IA represents out-of-phase bursting activity for $G_{E}=0.05$ and the region labeled as IP represents intermittent behavior with both EN's showing very long and simultaneous bursts observed in $G_{E}=-0.02$.

$$
\left(1-S_{\infty}\right) \tau_{s} \frac{d S(t)}{d t}=\left(S_{\infty}-S(t)\right)
$$

where

$$
S_{\infty}\left(x_{\text {pre }}\right)=\tanh \left[\frac{x_{\text {pre }}-x_{\text {th }}}{x_{\text {slope }}}\right],
$$

when $x_{\text {pre }}>x_{\text {th }}$. Otherwise $S_{\infty}\left(x_{\text {pre }}\right)=0$.

$G_{c}$ is the maximal synaptic conductance, $S(t)$ the instantaneous synaptic activation, $S_{\infty}$ the steady-state synaptic activation, $x_{\text {rev }}$ the synaptic reversal potential, and $x_{\text {pre }}$ and $x_{\text {post }}$ are the presynaptic and postsynaptic voltages, respectively. $\tau_{s}$ is the synaptic time constant, $x_{\text {th }}$ the synaptic threshold voltage, and $x_{\text {slope }}$ the synaptic slope voltage.

The synaptic reversal potentials were selected so that the currents injected into the postsynaptic EN's were always negative for inhibitory synapses and positive for excitatory synapses, emulating the biological synapses [16]. The synaptic threshold voltages were set in the middle of the amplitude of the bursts, and the synaptic slope voltage was adjusted to make the output of the hyperbolic tangent slightly saturated at the spikes. In our experiments $G_{c}$ was varied as we collected different data sets. We used standard values for the other parameters in the dynamic clamp program: $x_{\mathrm{rev}}=-80$ $\mathrm{mV}$ (inhibitory synapses) or $x_{\text {rev }}=-20 \mathrm{mV}$ (excitatory synapses); $\tau_{s}=10 \mathrm{~ms} ; x_{\text {th }}=-50 \mathrm{mV}$; and $x_{\text {slope }}=10 \mathrm{mV}$. As before we waited for at least $40 \mathrm{~s}$ after connecting the EN's with the chemical synapses before starting the recording of the $20 \mathrm{~s}$ of data from the membrane potential of the EN's.

\section{EXPERIMENTS}

To analyze the degree of synchronization of slow bursts between two coupled neurons (electrically or chemically) we proceed in the same manner as we used for our experiments on synchronized living neurons [6]. This was based on a method developed for the experimental studies of synchronization of chaotic oscillations in electronic circuits [19-21]. We used an overlap-add method of finite impulse response with a Hamming window, and used a fast Fourier transform and a cutoff frequency of $5 \mathrm{~Hz}$ to suppress the spikes, obtaining the filtered data series $x_{i}^{f}(t) ; i=1,2$. The synchronization of the EN's is quantified by calculating the difference $x_{d}^{f}(t)=x_{1}^{f}(t)-x_{2}^{f}(t)$, and studying the normalized standard deviation $\sigma_{N}=\sigma_{x_{d}^{f}} / \sigma_{x_{1}^{f}}$ and the normalized maximal deviation $\Delta_{N}=\left|x_{d}^{f}\right|^{\max } /\left(x_{1}^{f, \max }-x_{1}^{f, \min }\right)$ as a function of $G_{E}$ for the electrical coupling or as a function of $G_{c}$ for the chemical coupling. For notational convenience, we indicate excitatory couplings with values of $G_{c}>0$ and inhibitory couplings with values of $G_{c}<0$.

\section{A. Isolated neurons}

The parameters of the isolated neurons were set in the chaotic spiking-bursting regime. An example of the behavior of an isolated EN is shown in Fig. 3. Note that the scale for $x$ is double that of the numerical simulations shown in Fig. 1 because of the rescaling time constant in the analog integrator (units have dimensions in the analog implementation of the model, see Sec. III). The relative behavior of the spikes and slow oscillations can be seen in the plots of $x_{2}$ vs $x_{1}$ [Fig. 3(A)] and $x_{2}^{f}$ vs $x_{1}^{f}$ [Fig. 3(B)], respectively.

\section{B. Electrical coupling between two EN's}

We began with electrical coupling between two 4D ana$\log$ circuit models implementing Eq. (1). We varied only $G_{E}$ keeping all other parameters fixed. A convenient representation of the range of behavior we observed is presented in Fig. 4. Here, overlying values of $\sigma_{N}\left(G_{E}\right)$ and $\Delta_{N}\left(G_{E}\right)$, we give a verbal description of the quantitative behavior of time series in each regime. To illustrate the phenomena seen in each regime $G_{E} \in[-1,1]$ of Fig. 4 we show examples of the time series for the membrane potentials $x_{1}(t), x_{2}(t)$ of the two neurons in Figs. 5 and 6. 

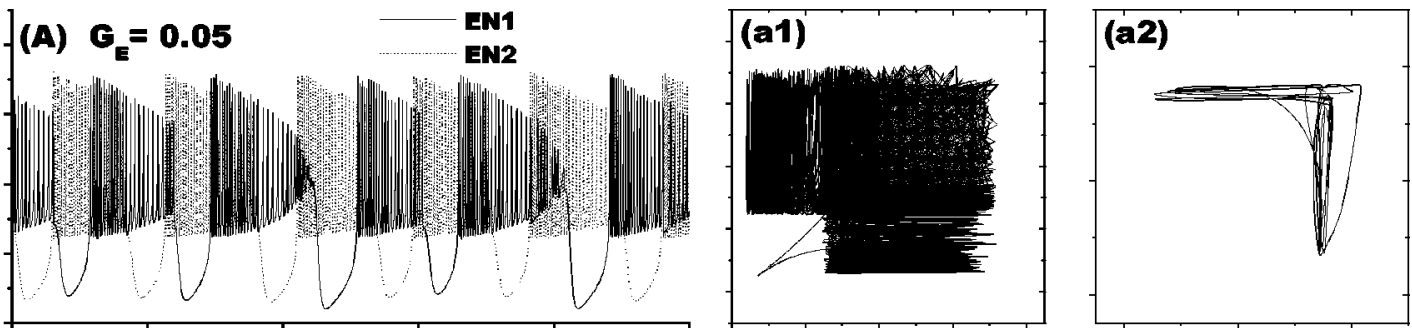

(B) $G_{E}=0.1$
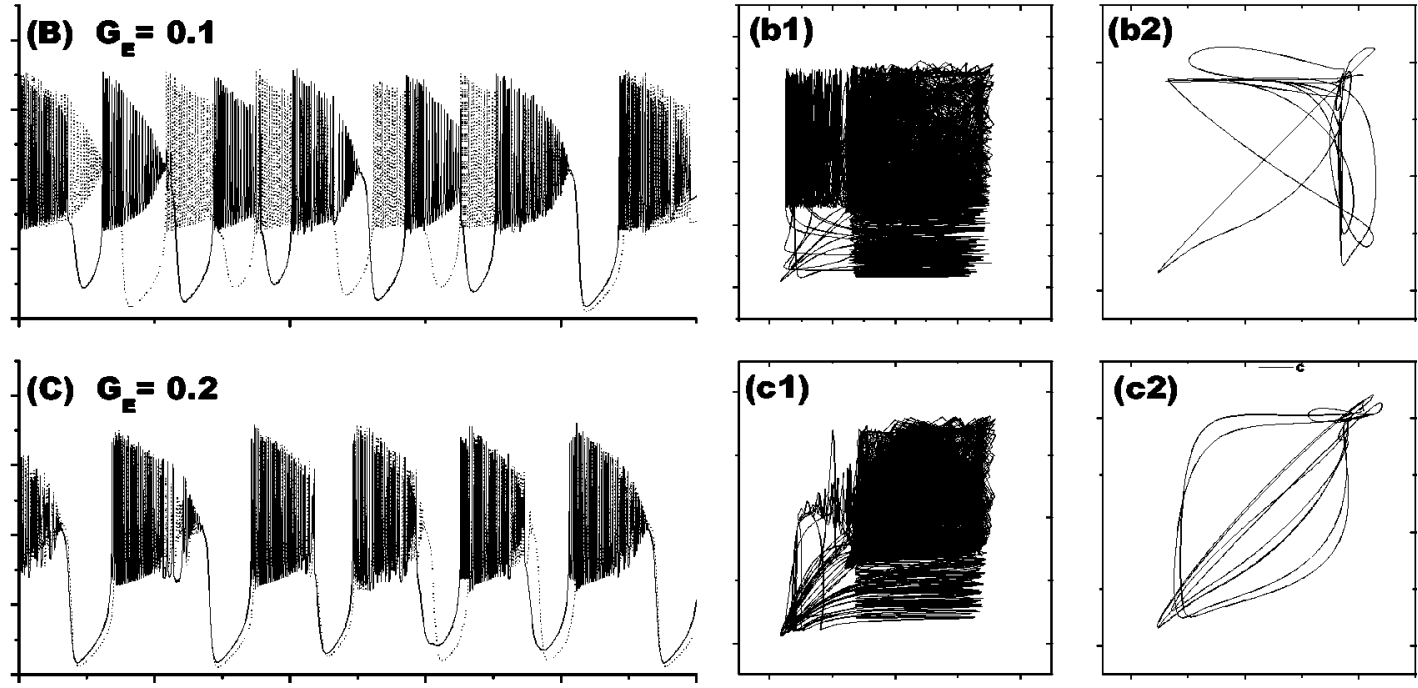

(D) $G_{-}=0.3$
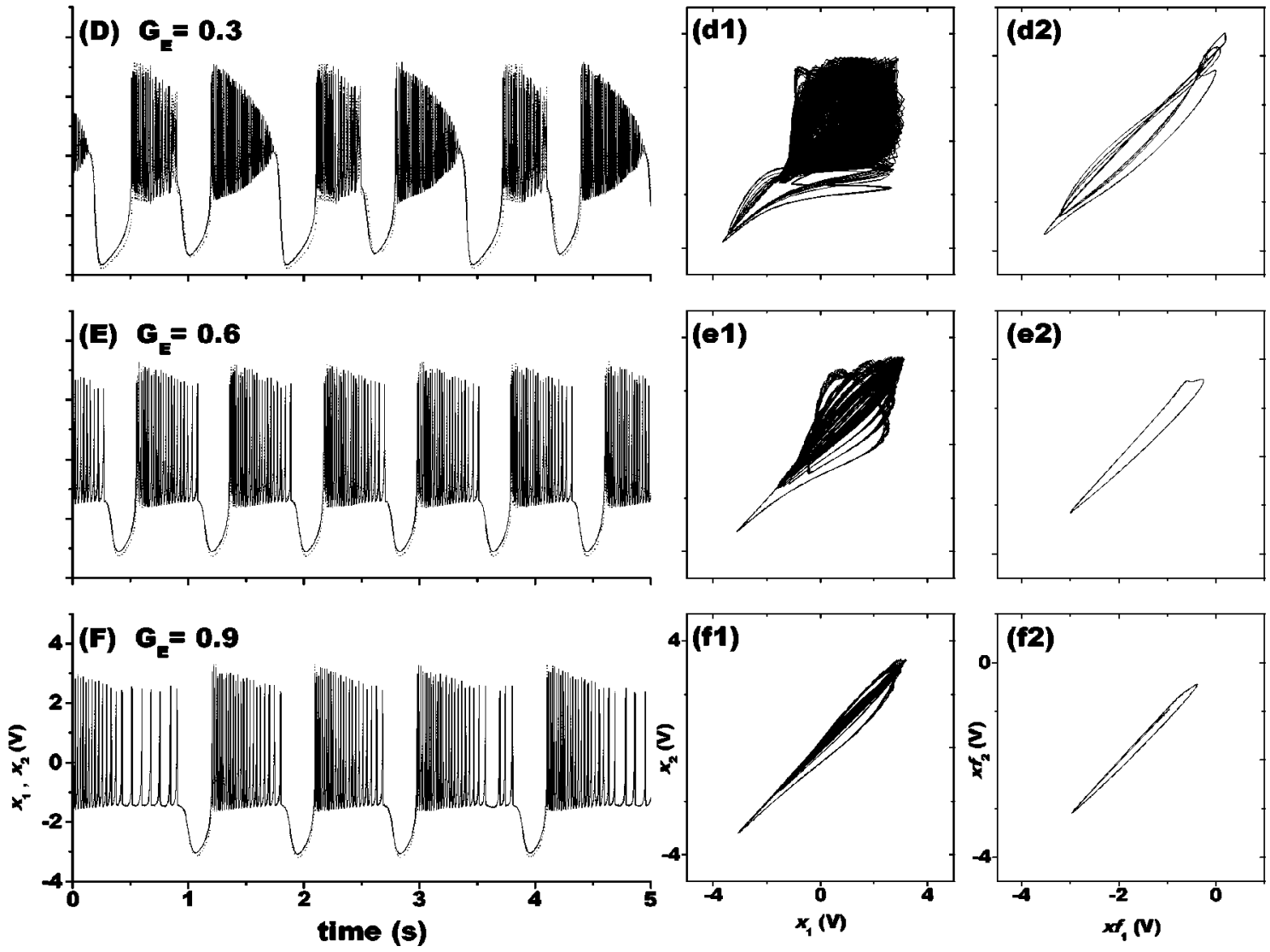

FIG. 5. Positive electrical coupling of two chaotic EN's. Characteristic time series of the membrane potentials $x_{1}(t), x_{2}(t)[(\mathrm{A})-(\mathrm{F})]$ as we vary $G_{E}$. Phase portrait $x_{2}(t)$ vs $x_{1}(t)[(\mathrm{a} 1)-(\mathrm{f} 1)]$. Phase protraits after 20-Hz low-pass filtering $x_{2}^{f}(t)$ vs $x_{1}^{f}(t)[(\mathrm{a} 2)-(\mathrm{f} 2)]$. (A) $G_{E}$ $=0.05$ intermittent out-of-phase bursting activity. (B) $G_{E}=0.1$ nearly independent chaotic spiking-bursting pattern. $(\mathrm{C}) G_{E}=0.2$ chaotic oscillations with most bursts synchronized. (D) $G_{E}=0.3$ periodic oscillations with partial synchronization of the EN's, the spikes are not synchronized. (E) $G_{E}=0.6$ periodic oscillations with the complete synchronization of the EN's. (F) $G_{E}=0.9$ chaotic but completely synchronized oscillations. 


\section{Results for $0 \leqslant G_{E} \leqslant 1$}

(i) When $G_{E} \approx 0.0$ the two neurons are uncoupled and display independent chaotic oscillations as shown in Fig. 3.

(ii) For small, positive coupling $0.0<G_{E}<0.2$, regions of nearly independent chaotic spiking-bursting activity are observed as well as some regions of synchronized bursting activity as shown in Fig. 5(B) where we set $G_{E}=0.1$. There is a small range of $G_{E}\left(G_{E} \approx 0.05\right)$ in which intermittent antiphase bursting behavior can be found. The burst length in this case is kept nearly regular from burst to burst as shown in Fig. 5(A).

(iii) For $0.2 \leqslant G_{E}<0.3$ the behavior is still chaotic for the two neurons but most of the bursts are synchronized as shown in Fig. 5(C) where we set $G_{E}=0.2$.

(iv) From $0.3 \leqslant G_{E}<0.8$ the bursting activity becomes regular going from a region in which there is partial synchronization (spikes not synchronized), as shown in Fig. 5(D) where we set $G_{E}=0.3$, to a region of total synchronization (bursts and spikes synchronized), shown in Fig. 5(E) where we set $G_{E}=0.6$.

(v) From $0.8 \leqslant G_{E}<1.0$ there is total synchronization in the spiking-bursting activity, and the oscillations are chaotic as shown in Fig. $5(\mathrm{~F})$ where we set $G_{E}=0.9$.

\section{Results for $-1 \leqslant G_{E} \leqslant 0$}

For negative coupling $G_{E}<0$, the oscillations are predominantly chaotic and the hyperpolarizing regions, where the membrane voltage is quite negative, of the signals are all in antiphase. The average burst length decreases as the coupling becomes stronger as shown in Fig. 6. For a small range of $G_{E}\left(G_{E} \approx-0.02\right)$ very long bursts were observed as shown in Fig. 6(A).

\section{Comparison of coupled EN's with electrically coupled biological neurons}

$\sigma_{N}\left(G_{E}\right)$ and $\Delta_{N}\left(G_{E}\right)$ provide quantitative measures of the synchronization between two EN's. In our report on the experimental work [6] with two biological cells, the results for $\sigma_{N}\left(G_{E}\right)$ and $\Delta_{N}\left(G_{E}\right)$ can be seen in Fig. 5 of that paper. Note that, as in the case of coupled biological neurons, we have here a bifurcation between positive and negative electrical coupling. In the experimental work on electrically coupled biological neurons a value for the external coupling $g_{a} \approx-200 \mathrm{~ns}$ serves to null out the natural coupling of about that amount, so the figures here and in the earlier paper are to be compared by sliding $G_{E}=0$ here to $g_{a} \approx-200$ ns there. Both in the biological and electronic experiments, the sharp phase transition from very small $\sigma_{N}, \Delta_{N}$ for positive coupling to large, nearly constant values is associated with the rather rapid change from nearly and then fully synchronous behavior for positive couplings to out-of-phase oscillations for negative couplings.

The $\sigma_{N}\left(G_{E}\right)$ and $\Delta_{N}\left(G_{E}\right)$ curves in the paper on coupled biological neurons [6] shows far fewer points and consequently less detail that our curves for coupled 4D EN's. Clearly this is because of the resolution in the biological experiments and the difficulty in performing experiments at such closely chosen values of $G_{E}$. At this time the details of behavior revealed in the present experiments on EN's have not been verified in the biological setting. One should view our Fig. 4 and Fig. 5 of Ref. [6] as in excellent qualitative agreement.

\section{Chemical synapses between two EN's}

We have observed the behavior of two 4D EN's coupled with identical chemical synapses. Two dynamic clamp versions of chemical synapses, based on the integration of Eq. (5), were built with identical parameters and then used to couple two 4D EN's. We then varied $G_{c}$ in each chemical synapse over the range $0 \leqslant G_{c} \leqslant 200 \mathrm{~ns}$ for an excitatory synapse, namely $x_{\text {rev }}=-20 \mathrm{mV}$, and over the range $0 \leqslant G_{c}$ $\leqslant 500 \mathrm{~ns}$ for an inhibitory synapse, namely, $x_{\text {rev }}=-80 \mathrm{mV}$. The other parameters were held fixed at $\tau_{s}=10 \mathrm{~ms}, x_{\mathrm{th}}=$ $-50 \mathrm{mV}$, and $x_{\text {slope }}=10 \mathrm{mV}$. In Fig. 7 we collect the statistical results, expressed in our usual quantities $\sigma_{N}\left(G_{c}\right)$ and $\Delta_{N}\left(G_{c}\right)$, for both excitatory and inhibitory synaptic connections. Negative values of $G_{c}$ represent inhibitory connections. This, perhaps apparently peculiar method of presentation allows us to see immediately the relationship between excitatory and inhibitory interconnections. As earlier with electrical couplings we provide a verbal description of each region of behavior over the whole range of $G_{c}$. We show examples of the time series for the membrane potential $x$ of the two neurons in Figs. 8 and 9.

\section{Excitatory chemical synapses}

When coupled with implementations of excitatory chemical synapses the EN's displayed the following behaviors:

(i) When $G_{c} \approx 0$ ns the two neurons are uncoupled and display independent chaotic oscillations as shown in Fig. 3.

(ii) For positive coupling $0<G_{c}<100 \mathrm{~ns}$ a transition from the chaotic behavior to regular spiking/bursting is observed. For small coupling the independent chaotic spiking/ bursting activity of the uncoupled neurons is replaced by a behavior in which most of the bursts are synchronized, but the oscillations are still chaotic as shown in Fig. 8(A) for $G_{c}=10$ ns. As $G_{c}$ is increased all the bursts become synchronized, and the activity becomes periodic as shown in Fig. 8(B) for $G_{c}=100 \mathrm{~ns}$.

(iii) For $G_{c}>100 \mathrm{~ns}$ the bursts remain synchronized and become longer, but there are no longer any spikes during the ending part of the bursts as shown in Fig. 8(C) for $G_{c}$ $=200 \mathrm{~ns}$.

\section{Inhibitory chemical synapses}

Finally we report on our experiments with an electronic version of an inhibitory chemical synapse. This inhibitory synaptic coupling occurs in the lobster central pattern generator (CPG) as well as many other CPGs, and we have suggested [2] that inhibitory chemical coupling will lead to regularization of the chaotic oscillations of the individual neurons.

(i) For small $G_{c}$ the oscillations are still chaotic, but all of the hyperpolarizing regions of the membrane voltages are in antiphase as shown in Fig. 9(A) for $G_{c}=8 \mathrm{~ns}$.

(ii) When $G_{C} \approx 20 \mathrm{~ns}$ the oscillations become periodic, and all the hyperpolarizing regions are in out-of-phase as shown in Fig. 9(B).

(iii) For $25 \mathrm{~ns} \leqslant G_{c}<50 \mathrm{~ns}$ the out-of-phase behavior of the hyperpolarizing regions remains, but the oscillations are 

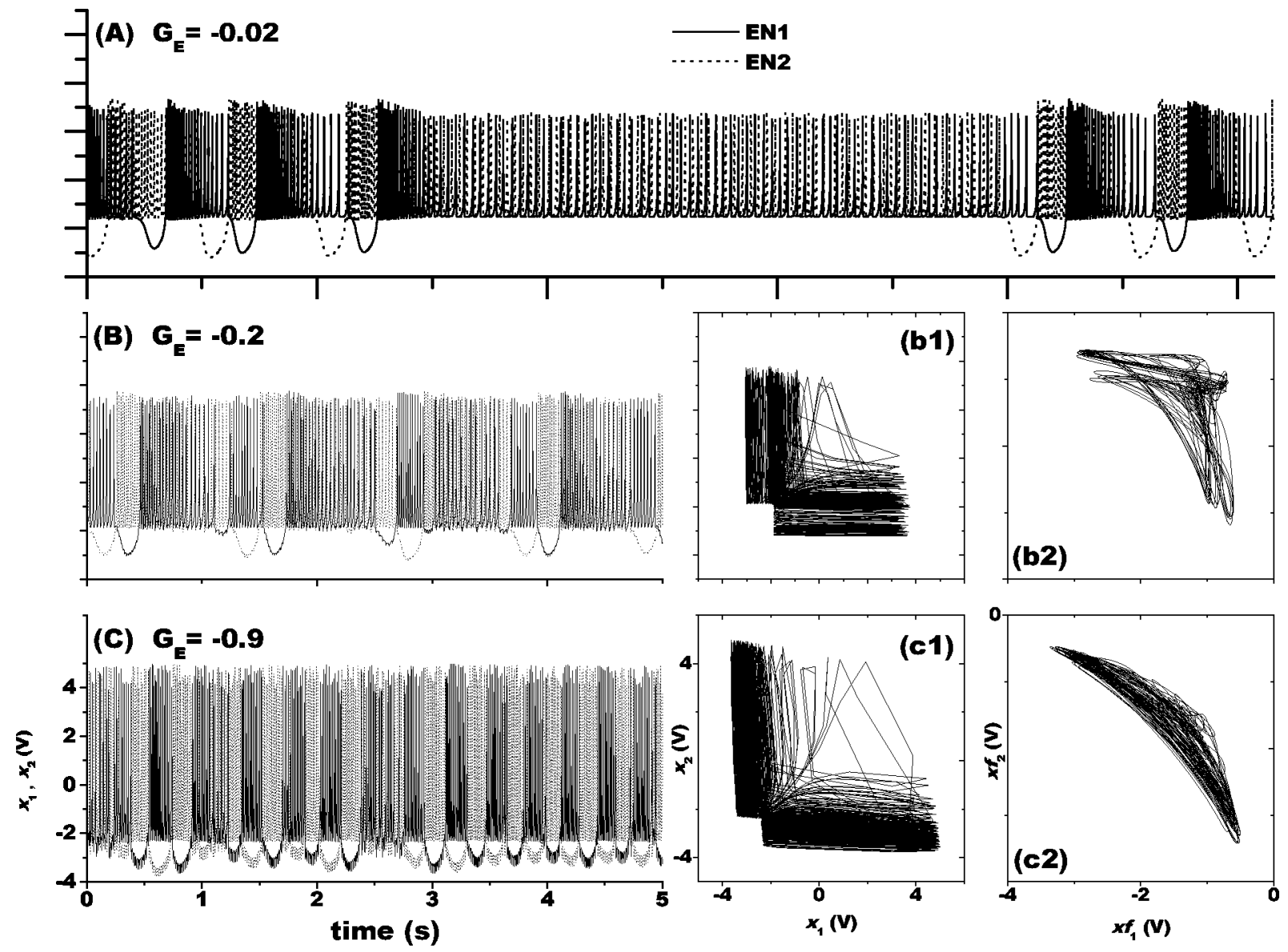

FIG. 6. Negative electrical coupling of two chaotic EN's. Characteristic time series of the membrane potentials $x_{1}(t), x_{2}(t)[(\mathrm{A})-(\mathrm{C})]$ as we vary $G_{E}$. Phase portraits of $x_{2}(t)$ vs $x_{1}(t)$ [(b1) and (c1)]. Phase portraits after 20-Hz low-pass filtering $x_{2}^{f}(t)$ vs $x_{1}^{f}(t)$ [(b2) and (c2)]. (A) $G_{E}=-0.02$ intermittent simultaneous long bursts in the two EN's. (B) $G_{E}=-0.2$ chaotic out-of-phase spiking-bursting behavior. (C) $G_{E}=-0.9$ fast chaotic out-of-phase spiking-bursting behavior. The time scale used in the time series plot is the same for all examples.

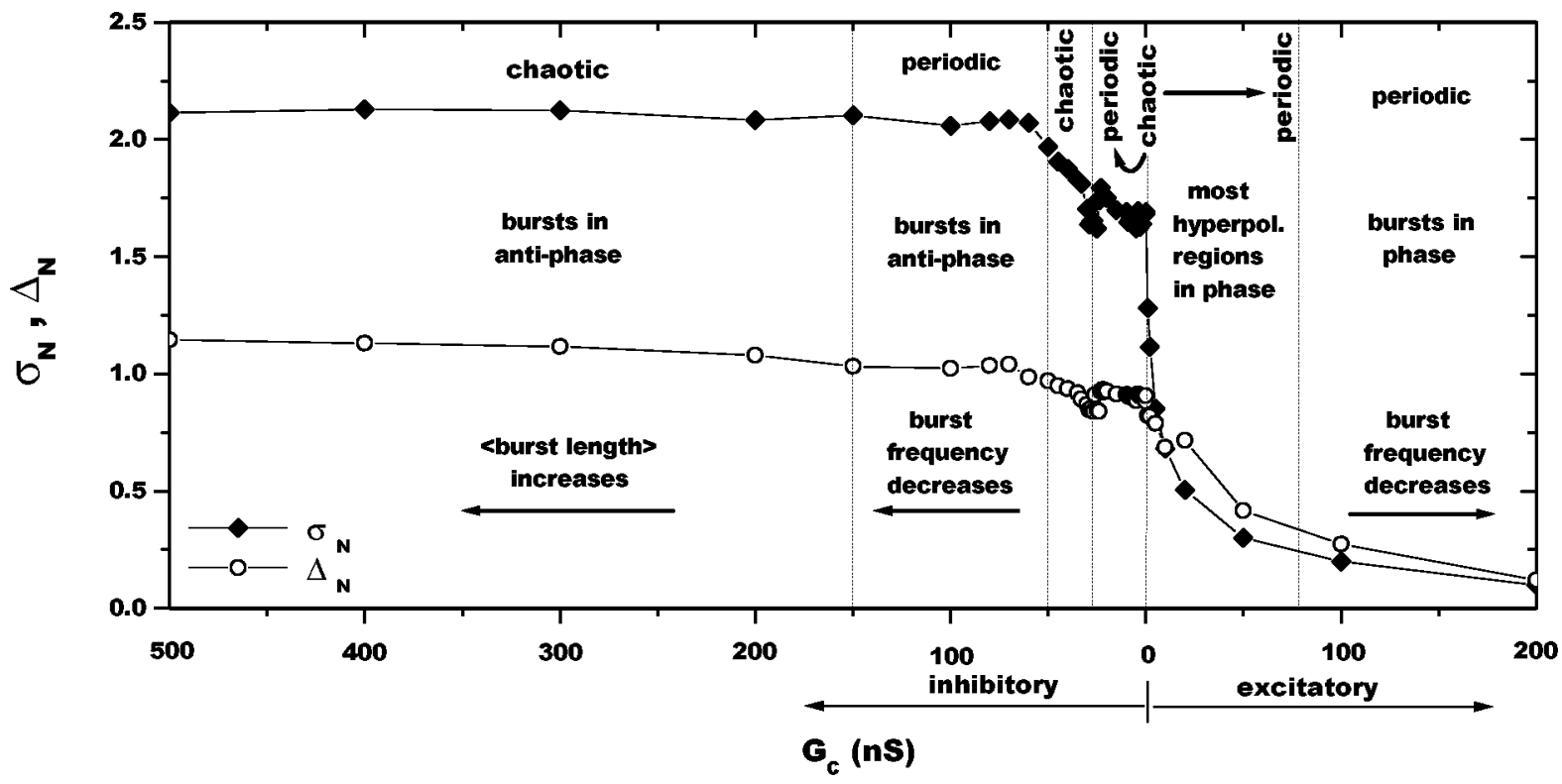

FIG. 7. Normalized standard deviation $\sigma_{N}\left(G_{c}\right)$ and normalized maximal deviation $\Delta_{N}\left(G_{c}\right)$ computed after 20-Hz low-pass filtering in the membrane potential of two EN's coupled with identical chemical synapses for different values of the synaptic conductance $G_{c}$ for both excitatory $\left(G_{c}>0\right)$ and inhibitory $\left(G_{c}<0\right)$ connections. For very small excitatory coupling $\left(G_{c} \approx 0\right)$ the behavior of the EN's is nearly independent and chaotic. 

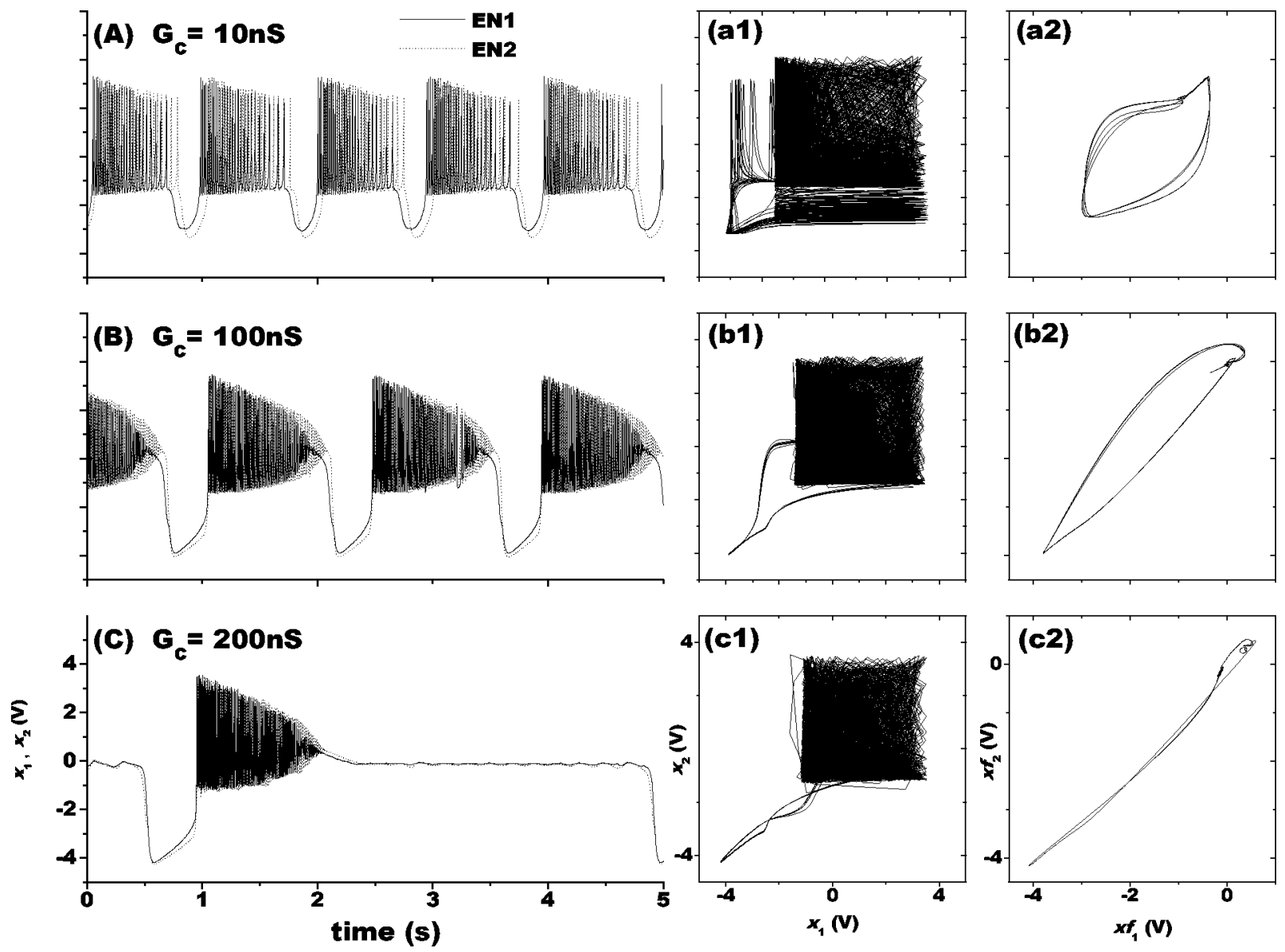

FIG. 8. Excitatory chemical coupling of two chaotic EN's. Characteristic time series of the membrane potentials $x_{1}(t), x_{2}(t)[(\mathrm{A})$ to $(\mathrm{C})]$. Phase portraits $x_{2}(t)$ vs $x_{1}(t)$ [(a1)-(c1)]. Phase portraits after 20-Hz low pass filtering $x_{2}^{f}(t)$ vs $x_{1}^{f}(t)[(\mathrm{a} 2)-(\mathrm{c} 2)]$. (A) $G_{C}=10-\mathrm{ns}$ chaotic but nearly synchronized bursting behavior. (B) $G_{C}=100$-ns periodic and synchronized bursting activity of the EN's. (C) $G_{C}=200--n s$ periodic and synchronized activity with long bursts and spikes vanishing before the end of the bursts.

chaotic again as shown in Fig. $9(\mathrm{C})$ for $G_{c}=25 \mathrm{~ns}$.

(iv) For $50 \mathrm{~ns} \leqslant G_{c}<150 \mathrm{~ns}$ the oscillations regularize again, and the behavior is periodic with out-of-phase bursting as shown in Fig. 9(D) for $G_{c}=50 \mathrm{~ns}$ and in Fig. 9(E) for $G_{c}=100 \mathrm{~ns}$.

(v) For $G_{c}>150 \mathrm{~ns}$ the oscillations are chaotic and long out-of-phase bursts are observed as shown in Fig. 9(F) for $G_{c}=300 \mathrm{~ns}$.

The only experiments we know which relate to these observations on two chemically coupled EN's are not a precise match, but bear noting. Elson [22] has isolated a pair of lateral pyloric (LP) and pyloric dilator (PD) neurons from the pyloric circuit of the CPG of the lobster STG; these have mutual inhibitory coupling. Elson varied the strength of the chemical coupling using neuromodulators and making measurements at four values of $G_{c}$ over a nominal rage of 20-60 ns. He observed only the behavior reported in the penultimate item of our experiments on inhibitory coupling. Unfortunately, control of the identity of the mutual inhibitory couplings was not possible, nor was it possible for us to directly compare the calibration of Elson's indication of the magnitude of $G_{c}$ with our own choices in using EN's. To date then, we have no direct laboratory evidence on synchronization of biological neurons mutually coupled with chemical synapses. This is in contrast to our observations on electrically coupled biological neurons [6]. This represents an interesting oppor- tunity for biological experiments which may be directly compared to our results using EN's.

\section{Coupling between electronic and living neurons}

We have previously reported experiments on replacing the anterior burster $(\mathrm{AB})$ neuron from the Pyloric CPG in its interaction with an isolated pair of PD neurons with a 3D EN [14]. For completeness in light of the work reported in this paper, we carried out an experiment in which one of our 4D neurons was coupled bidirectionally to one of the PD neurons in the $\mathrm{AB} / \mathrm{PD}$ pacemaker group of the Pyloric CPG. The full description of the methods used in the biological preparation will appear elsewhere [23], but here we quite briefly summarize those points important to the main thrusts of this article.

These experiments were carried out on one of the two PD neurons from the Pyloric CPG of the lobster [24]. The STG of the California spiny lobster, Panulirus interruptus, was removed using standard procedures and pinned out in a dish lined with silicone elastomer and filled with normal lobster saline. The STG was isolated from its associated anterior ganglia, which provide activating inputs, by cutting the stomatogastric nerve. Two glass microelectrodes were inserted in the soma of the PD neuron: one for intracellular voltage recording and another one for current injection. The voltage 

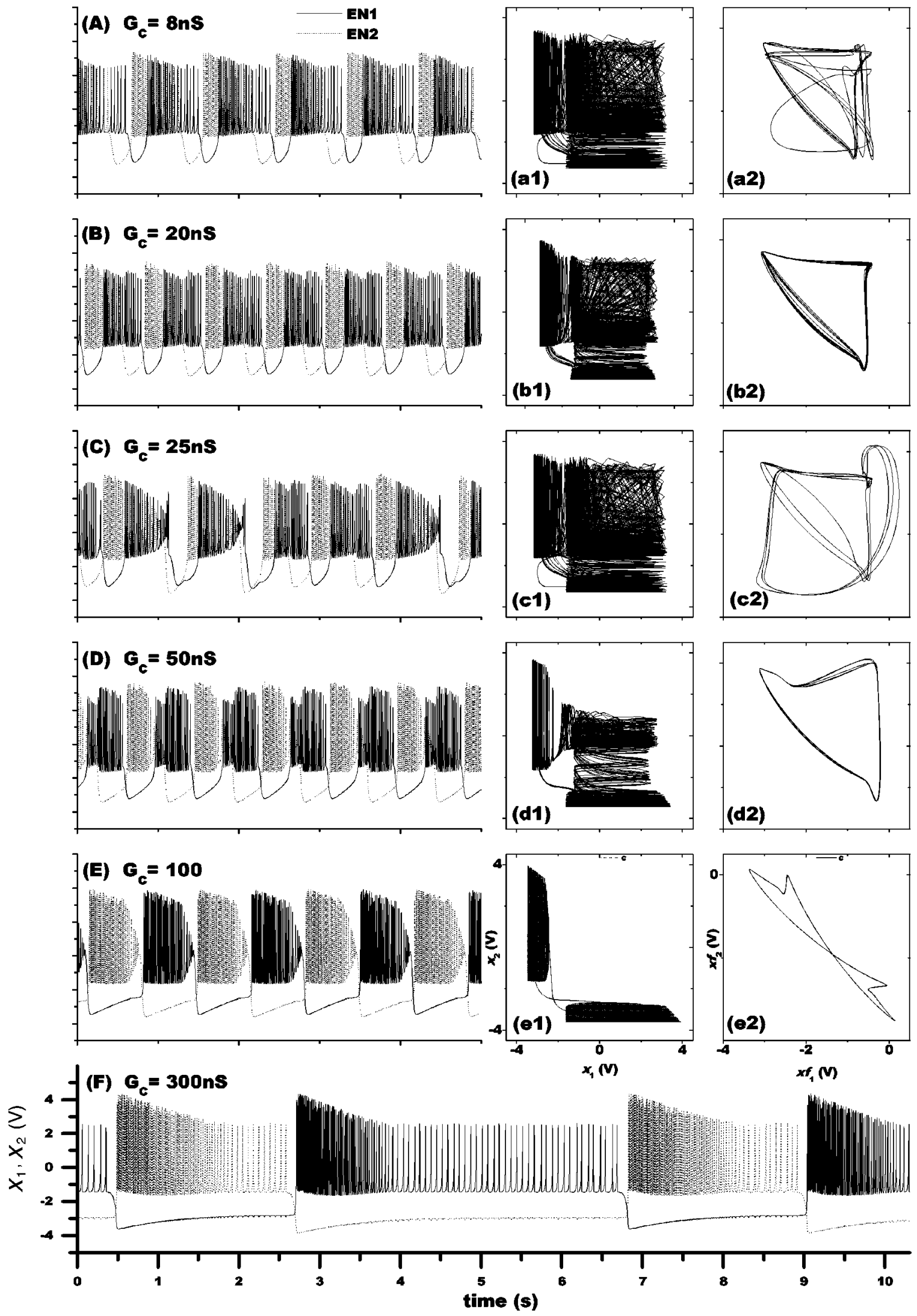

FIG. 9. Inhibitory chemical coupling of two chaotic EN's. Characteristic time series of the membrane potentials $x_{1}(t), x_{2}(t)[(\mathrm{A})-(\mathrm{F})]$. Phase portraits $x_{2}(t)$ vs $x_{1}(t)$ [(a1)-(f1)]. Phase portraits after 20-Hz low-pass filtering $x_{2}^{f}$ vs $x_{1}^{f}[(\mathrm{a} 2)-(2 \mathrm{f})]$. (A) $G_{C}=8$-ns chaotic oscillations with all hyperpolarizing regions out-of-phase. (B) $G_{C}=20$-ns periodic pattern with hyperpolarizing regions out-of-phase and some burst superposition. (C) $G_{C}=25$-ns chaotic oscillations. (D) $G_{C}=50$-ns periodic out-of-phase bursting behavior with some burst superposition. (E) $G_{C}=100$-ns periodic out-of-phase spiking-bursting behavior. (F) $G_{C}=300$-ns chaotic out-of-phase spiking-bursting pattern. The time scale used in the time series plot is the same for all examples. 

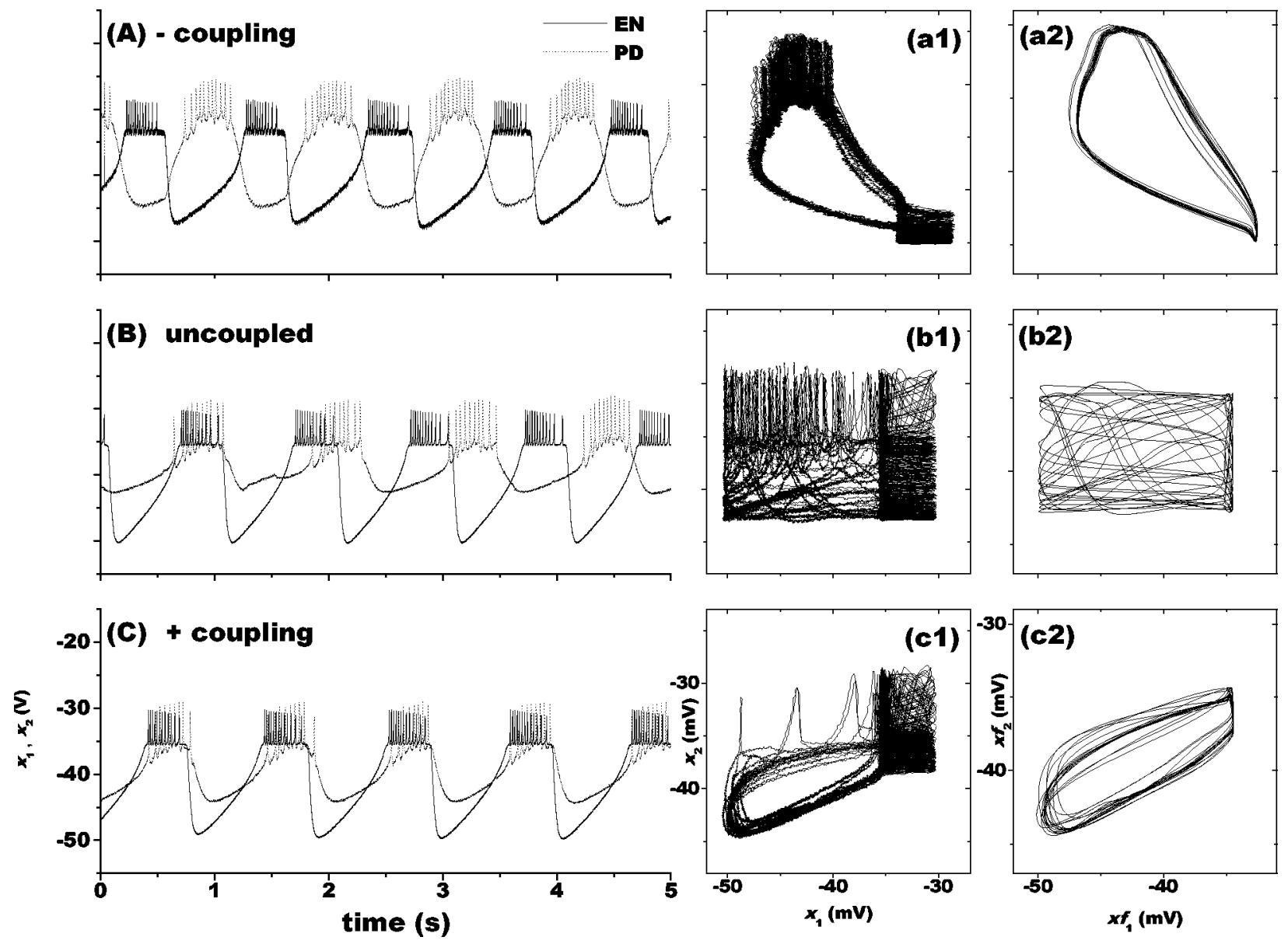

FIG. 10. Electrical coupling of an EN and a living PD neuron from the STG of the lobster Panulirus interruptus. Characteristic time series of the membrane potentials $x_{1}(t), x_{2}(t)$ [(A)-(C)]. Phase portraits of $x_{2}(t)$ vs $x_{1}(t)$ [(a1)-(c1)]. Phase portraits after 20-Hz low-pass filtering $x_{2}^{f}(t)$ vs $x_{1}^{f}(t)$ [(a2)-(c2)]. (A) Negative coupling-out-of-phase bursting activity. (B) Uncoupled neurons. (C) Positive electrical coupling-synchronized bursting behavior

signals were digitized at 10000 samples/s. The two PD neurons remained coupled to each other and to the $A B$ neuron by their natural electrical synapses, but were isolated from the rest of the CPG by blocking chemical input synapses with picrotoxin $(7.5 \mu \mathrm{M})$. The artificial electrical coupling was provided by injecting in the EN and in the PD opposite currents. More details of the experimental setup can be found in Ref. [14]. The membrane voltage of the EN was reshaped to make its amplitude ratio in spiking/bursting mode, its total amplitude, and its voltage offset similar to those of the PD neuron. Only electrical coupling, positive and negative, is reported here.

We connected the neurons with the analog electrical synapse and observed their spiking-bursting behavior as shown in Fig. 10. When uncoupled, the neurons had independent spiking/bursting activity as shown in Fig. 10(B). For large enough negative coupling the neurons are synchronized and fire out-of-phase as shown in Fig. 10(A). For positive coupling the neurons show synchronized bursting activity as shown in Fig. 10(C). For this value of $G_{E}$ the bursts are synchronized but not the spikes.

This result is in agreement with the experiments made with a pair of electrically coupled EN's, as we discussed above, as well as for a pair of living STG neurons [6].

\section{DISCUSSION}

The EN's described in this paper are simple analog circuits which integrate 4D differential equations representing fast and slow subcellular processes that give rise to the characteristic spiking and spiking-bursting behavior of CPG neurons. The EN's are also able to reproduce the dynamical bifurcations seen in the living neurons. The performance of the 4D EN's, compared with the earlier 3D version, showed a richer behavior with larger regions of parameter space in which we observed chaotic behavior. The 4D EN's were also easier to tune in order to reproduce the behavior of living CPG cells; in particular, we can tune the width of the bursts before setting the EN in the chaotic regime. This study comprises: (a) two electrically coupled EN's and (b) two EN's connected with excitatory and inhibitory chemical synapses. These two types of connections exist in almost all known CPGs. The range of observations summarized in Figs. 4 and 7 shows the rich behavior and complexity of these minimal network configurations. It indicates how small changes in the coupling conductance can drive the cells into completely different regimes. In particular, some of our experiments predict the appearance of chaotic out-of-phase synchronization for different coupling configurations. These results are displayed in Figs. 6(C) and 9(F). In general, the experiments with the 
EN's contribute directly to our understanding of the origin of regularization of individually chaotic neurons through cooperative activity.

Although the EN's presented here reproduce many characteristics of the dynamical behavior for the living cells, they fail to completely reproduce several aspects of their activity. In particular, the shape of the spikes in the EN's is not exactly the same as the one observed in the living neurons. Also, the EN's do not show the adaptation characteristics of the biological neurons due to plastic changes in the conductances of the cell membrane, since the model does not include any mechanism to include these phenomena.

How complicated should one require a model neuron to be? In our view the answer depends on the neural function one wishes to represent. The analysis of the electrical activity of isolated neurons from the lobster Pyloric CPG indicates that the number of active degrees of freedom is not very large, ranging from three to five in various environments, and this suggests a very simple representation in terms of dynamical equations. Our analysis [8] of much richer $\mathrm{HH}$ models of these individual neural oscillators also indicates that in the regime of biological operation, the number of active degrees of freedom is equally small. On this basis we developed the HR type models of these neurons both in numerical simulation and in analog electrical circuitry.

This paper has moved that inquiry about the complexity of representation for the components of a biologically realistic neural network to another level. Here we have investigated whether the simplified neural models, when coupled together in small networks but in biologically realistic manners, can reproduce our observations of biological neurons alone. The striking result of the observations presented here, when the experimental setup matches that of the biological networks, is that the observed behavior of the EN's also matches. Further, using our EN's, we are able to make distinct predictions about the behavior of biological or hybrid (biological and EN) networks in settings not yet investigated.

Our experiments on coupled biological neurons and EN's provide further ground for testing the validity of numerical and electronic models of individual neural behavior as well as presenting interesting new examples of coupled nonlinear oscillators. Hybrid circuits with biological and electronic neurons coupled together are a powerful mechanism for understanding the modes of operation of CPG's. The hybrid system constitutes an easy way to change the connectivity and global topology of the CPG. The roles of intrinsic dynamics of the neurons and the synaptic properties of the network in rhythm generation can be easily studied with these hybrid configurations [14].

There have been previous efforts to study electronic neurons alone and in conjunction with biological neurons. An early example is the work of Yarom [25] where a network of four oscillators, realized as an analog circuit, was interfaced with an olivary neuron in a slice preparation. Yarom studied the response of the olivary neuron when it received oscillating electrical input from the network. There was no feedback from the biological neuron to the network he constructed. Le Masson et al. [26] developed a digital version of a neuron comprising a $\mathrm{HH}$ [7] model of various pyloric CPG neurons with three compartments and eight different ion channels which ran on a DSP board located on the bus of a personal computer. They connected this model into a variety of different configurations of subcircuits of the pyloric CPG replacing at various times the LP, a PD, or a pyloric neuron. Using this "hybrid" setup they verified that many aspects of the pyloric rhythm are accurately reproduced when their DSP based neuron replaces one of the biological neurons in their system. In subsequent work [27,28], this group has developed very large scale integrated (VLSI) devices for integrating the $\mathrm{HH}$ models and has utilized them in mixed circuits (EN's and biological neurons), replacing the DSP version of the conductance models in their biological preparations. The complexity of these EN's has not been needed in our modeling nor in the further experiments on their interaction with each other as reported here. We have not found any reports in the literature on the mutual interaction of these analog VLSI neural circuits.

There are two interesting directions to which the results reported here may point:

(1) Biologically realistic neural networks of much greater size than the elementary ones investigated here may be efficiently investigated numerically or in analog circuitry using the realistic, but simple HR type models. The integration of the model equations is no challenge to easily available computing power and large networks should be amenable to investigation and analysis.

(2) The networks investigated here are subcircuits of a biological circuit of about 15 neurons which has the functional role of a control system: commands are presented from other ganglia of the lobster and this Pyloric circuit must express voltage activity to the muscles to operate a pump for shredded food passing from the stomach to the digestive system. Many other functions are asked of biological neural networks. Using the full richness of $\mathrm{HH}$ models for the component neurons may seem attractive at one level, but the results presented here suggest that many interesting questions may be asked of those networks using the simplified component neurons studied here.

\section{ACKNOWLEDGMENTS}

R.D. Pinto was supported by the Brazilian Agency Fundação de Amparo à Pesquisa do Estado de São Paulo FAPESP. P.V. acknowledges support from MEC. Partial support for this work came from the U.S. Department of Energy, Office of Science, under Grants Nos. DE-FG0390ER14138 and DE-FG03-96ER14592. We also acknowledge the many conversations we have had with Ramon Huerta, Rob Elson and Allen Selverston on the dynamics of CPG neurons. 
[1] L. Glass and M. C. Mackey, From Clocks to Chaos: the Rhythms of Life (Princeton University Press, Princeton, NJ, 1988).

[2] H. D. I. Abarbanel, R. Huerta, M. I. Rabinovich, P. F. Rowat, N. F. Rulkov, and A. I. Selverston, Neural Comput. 8, 1567 (1996)

[3] H. D. I. Abarbanel, M. B. Kennel, M. Buhl, and C. Lewis, Phys. Rev. A 60, 2360 (1999).

[4] R. Roy and G. VanWiggeren, Science 279, 1198 (1998).

[5] M. I. Rabinovich and H. D. I. Abarbanel, Neuroscience 87, 5 (1998).

[6] R. Elson, R. Huerta, N. F. Rulkov, H. D. I. Abarbanel, and M. I. Rabinovich, Phys. Rev. Lett. 81, 5692 (1998).

[7] A. L. Hodgkin and A. F. Huxley, J. Physiol. (London) 117, 500 (1952).

[8] M. Falcke, R. Huerta, M. I. Rabinovich, H. D. I. Abarbanel, R. C. Elson, and A. I. Selverston, Biol. Cybern. 82, 517 (2000).

[9] M. I. Rabinovich, P. Varona, J. J. Torres, R. Huerta, and H. D. I. Abarbanel, Physica A 263, 405 (1999).

[10] P. Varona, J. J. Torres, H. D. I. Abarbanel, M. I. Rabinovich, and R. E. Elson (unpublished).

[11] H. D. I. Abarbanel, Analysis of Observed Chaotic Data (Springer, New York, 1996).

[12] H. Kantz and T. Schreiber, Nonlinear Time Series Analysis (Cambridge University Press, Cambridge, England, 1997).

[13] J. L. Hindmarsh and R. M. Rose, Proc. R. Soc. London, Ser. B 221, 87 (1984).

[14] A. Szücs, P. Varona, A. R. Volkovski, H. D. I. Abarbanel, M. I. Rabinovich, and A. I. Selverston, NeuroReport 11, 563 (2000).

[15] T. S. Parker and L. O. Chua. Practical Numerical Algorithms for Chaotic Systems (Springer, New York, 1989).

[16] Gordon M. Shepherd, Neurobiology, 3rd ed. (Oxford University Press, New York, 1994), see Chap. 7 for a discussion of these synaptic types.

[17] A. A. Sharp, L. F. Abbott, and E. Marder, J. Neurophysiol. 67, 1691 (1992).
[18] R. D. Pinto, A. Szücs, R. Elson, M. I. Rabinovich, and H. D. I. Abarbanel (unpublished).

[19] V. S. Afraimovich, N. N. Verichev, and M. I. Rabinovich, Radiophys. Quantum Electron. 29, 747 (1986).

[20] J. F. Heagy, L. M. Pecora, and T. L. Carroll, Phys. Rev. E 50, 1874 (1994).

[21] N. F. Rulkov, A. R. Volkovskii, A. Rodriguez-Lozano, E. Del Rio, and M. G. Velarde, Int. J. Bifurcation Chaos Appl. Sci. Eng. 2, 669 (1992).

[22] R. C. Elson (private communication).

[23] A. Szücs (private communication).

[24] R. M. Harris-Warrick et al., in Dynamic Biological Networks: The Stomatogastric Nervous System, edited by R. M. HarrisWarrick et al. (MIT Press, Cambridge, MA, 1992).

[25] Y. Yarom, Neuroscience 44, 263 (1999).

[26] S. Renaud-Le Masson, E. Marder, G. Le Masson, and L. Abbott, Neural Information Processing Systems 5 (Morgan Kaufmann San Mateo, CA, 1993), pp. 813-819; G. Le Masson, S. Le Masson, and M. Moulins, Prog. Biophys. Mol. Biol. 64, 201 (1995)

[27] A. Laflaquiere, S. Le Masson, J. P. Dom, and G. Le Masson, in Proceedings of the International Conference on Neural Networks (ICNN'97), Houston, TX, June, 1997 (IEEE, New York, 1997), Vol. 2, pp. 882-7; D. Dupeyron, S. Masson, Y. Deval, G. Le Masson, and J.-P. Dom, in Proceedings of the Fifth International Conference on Microelectronics for Neural Networks and Fuzzy Systems, MicroNeuro96, Lausanne, Switzerland, February, 1996 (IEEE Compututer Society, Los Alamitos, CA, 1996), pp. 311-16; S. Le Masson, Y. Deval, G. Le Masson, J. Tomas, D. Dupeyron, and J. P. Dom, in Proceedings of the Fourth International Conference on Microelectronics for Neural Networks and Fuzzy Systems, Turin, Italy, September, 1994 (IEEE Computer Society, Los Alamitos, CA, 1994), pp. 202-6.

[28] An excellent introduction to the issues involved, the approximations employed, the methods of implementation as well as to the results of some experiments can be found in the dissertation of G. Le Masson available online at www.ixl-ubordeaux.fr/equipes-ixl/neurone/Publications/These_Gwendal 